\title{
Características sociodemográficas y clínicas de pacientes con nefritis lúpica. Barranquilla, Colombia
}

\author{
Gladys Gaviria-Garcia' ${ }^{1}$, Aída, Maidana de Zarza², Gustavo Aroca-Martinez ${ }^{3}$ \\ ${ }^{1}$ Universidad de la Costa. Barranquilla, Colombia \\ ${ }^{2}$ Universidad Nacional de Asunción. Paraguay \\ ${ }^{3}$ Universidad Simon Bolivar. Facultad Ciencias de la Salud. Barranquilla. Colombia
} Cómo referenciar este artículo/
How to reference this article:

\author{
Gaviria-García G, Maidana de Zarza A, Aroca- \\ Martínez G. Características sociodemográficas y \\ clínicas de pacientes con nefritis lúpica. Barranquilla, \\ Colombia. Mem. Inst. Investig. Cienc. Salud. 2018; \\ 16(2): $32-37$
}

\section{RE S U M E N}

La Nefritis Lúpica (NL) es una complicación relevante del paciente con lupus eritematoso sistémico (LES). Las condiciones socio demográficas pueden ser vulnerables a la aparición de la enfermedad?. Estudio cuantitativo, descriptivo y transversal. Población conformada por 22 pacientes con (NL) del programa de Nefrored del Caribe que asistieron a una institución prestadora de salud en la ciudad de Barranquilla, de enero de 2017 a enero de 2018. La muestra fue por conveniencia. La información se realizó a través de instrumento tipo escala de Likert, conformada por las variables: características socio demográficas (sexo, ubicación geográfica, estado civil), tiempo de diagnóstico de la enfermedad y toma del medicamento ordenado. El $96 \%$ era de sexo femenino y el $4 \%$ de sexo masculino, $27.3 \%$ estuvo entre edades de 34 y 45 años, el $54.5 \%$ era procedente de la ciudad de Barranquilla, y el $2.7 \%$ de los pacientes tenìn diagnóstico en un plazo de tiempo menor a los 2 años de haber presentado manifestaciones clínicas. Las condiciones sociodemograficas y clinicas de los pacientes con LES que desarrollan NL permitirán desarrollar acciones para un diagnóstico oportuno e intervención efectiva que coadyuven a la adherencia al tratamiento, y a reducir la morbilidad en los pacientes de la Region Caribe Colombiana.

Palabras clave: características de la población, nefritis lupica, lúpus eritematoso, características de los estudios epidemiológicos (DeCS Descriptores de Ciencia en Salud?).

\section{Sociodemographic and clinical characteristics of patients with Iupus nephritis. Barranquilla, Colombia}

\begin{abstract}
A B S T R A C T
Lupus Nephritis (LN) is a significant complication of the patient with systemic lupus erythematosus (SLE). Socio-demographic conditions may be vulnerable to the onset of the disease. This was a quantitative analytical and transversal study. Population comprised 22 patients with LN of the Renal Network of the Caribbean program attending a health care institution in the city of Barranquilla from January 2017 to January 2018. The sample was for convenience. The information was collected using a Likert scale instrument including these variables: socio-demographic characteristics (sex, geographical location, marital status), time of diagnosis of the disease, intake of medical prescription medicine. Ninety six percent was female and $4 \%$ male, $27.3 \%$ was between the ages of 34 and 45 years, 54.5\% was from the city of Barranquilla, and $2.7 \%$ patients were diagnosed in a period of time lower than 2 years of having presented clinical manifestations. The socio-demographic and clinical conditions of patients with SLE that developed NL will allow developing actions for a timely diagnosis and effective intervention for better adherence to treatment, and reduce the morbidity associated with socio-demographic conditions that may influence patients in the Colombian Caribbean Region.
\end{abstract}

Keywords: characteristics of the population, lupus nephritis, lupus erythematous, characteristics of epidemiological studies (DeCS Health Science Descriptors). 


\section{INTRODUCCIÓN}

Las enfermedades crónicas como la hipertensión arterial, alteraciones coronarias, enfermedad renal, diabetes mellitus, entre otras, continúan siendo la primera causa de muerte a nivel mundial, no obstante, datos publicados en 2008 revelan que la mortalidad por enfermedades coronarias han disminuido, mientras que la causada por enfermedades renales y la diabetes ha aumentado considerablemente en los últimos años en la mayor parte de los países Europeos y América Latina, aportando consigo altos costos al sistema sanitario a nivel mundial ${ }^{(1,2)}$.

Según datos de la Organización Mundial de la Salud (OMS), la diabetes mellitus (DM) acrecentará en las primeras décadas del siglo XXI en un $122 \%$, lo que representará pasar de la cifra actual de 135 millones de personas diabéticas a nivel mundial a una cantidad de 300 millones, lo cual coadyuvará a la aparición de enfermedades renales. Una de las complicaciones de la diabetes es la insuficiencia renal crónica (IRC), aunque en la actualidad, se dice que más de un tercio de los diabéticos, aún no han sido diagnósticados ${ }^{(3)}$.

Por otro lado, el lupus eritematoso sistémico (LES) es una enfermedad autoinmune caracterizada por el compromiso de múltiples órganos, siendo la Nefritis Lúpica (NL) una de las manifestaciones más graves. El LES es una de las enfermedades reumáticas sistémicas más frecuentes, con una incidencia creciente en Europa y América Latina, su prevalencia se estima en 9 por 10.000 habitantes, aunque el pronóstico ha mejorado muy lentamente en los últimos años, la calidad de vida de estos pacientes es visiblemente inferior a la población en general, y el riesgo de fallecer es de 2 a 3 veces mayor. Además, esta enfermedad conlleva altos costos al sistema sanitario, su pronóstico es complejo y su baja prevalencia hace necesario continuar realizando estudios de características genéticas y demográficas que evidencien la problemática ${ }^{(4,6)}$.

La prevalencia de LES en Colombia es de $(8,77 / 10.000)$ similar a la documentada en otros países de Latinoamérica, Argentina (6/10.000) a los calculados basados en la metodología COPCORD en Guatemala (1,2/10.000) y México (6/10.000) y Brasil $(9,8 / 10.000)$ por el mismo método. Así mismo se mantiene la tendencia de una mayor frecuencia de presentación en mujeres ${ }^{(7)}$.

Actualmente, se tienen examinados posibles factores de riesgo para el avance de esta enfermedad, la historia familiar es uno de los más fuertes, y es conocido que aproximadamente el $5 \%$ de todos los pacientes con enfermedades crónicas no trasmisibles, tienen síndromes hereditarios familiar. La incidencia de LES varía de acuerdo con las características de cada población, y estudios epidemiológicos muestran que existen diferentes esquemas de presentación de LES entre países. Asimismo, se han observado discrepancias entre grupos de población de la misma raza que viven en diferentes partes del mundo, lo que apoya que, además de susceptibilidad genética, los factores geográficos y ambientales están probablemente implicados en el desarrollo de esta enfermedad ${ }^{(8)}$.

La Nefritis Lúpica (NL) es la afectación visceral de gravedad que surge con más frecuencia en pacientes que se les diagnostica lupus eritematoso sistémico (LES), el daño renal,lleva a un deterioro irreversible, acrecentando la morbilidad y mortalidad de los afectados por LES, asímismo los pacientes con NL proliferativa tienen alto riesgo de morir o desarrollar insuficiencia renal terminal, mientras que los que tienen glomerulonefritis proliferativa focal manifiestan una longevidad del $72 \%$ y los afectados de glomerulonefritis proliferativa difusa, del $67 \%$ a los 5 años $^{(9-10)}$.

Por todo lo expuesto anteriormente, se desarrolló este estudio con el objetivo de describir algunos aspectos de las condiciones socio demográficas de los pacientes con Nefritis Lúpica (NL) de una institución prestadora de salud en la ciudad de Barranquilla.

\section{MATERIALES Y MÉTODOS}

Se diseñó un estudio cuantitativo, descriptivo, transversal. El universo conformado por 182 pacientes de la Región Caribe Colombiana adscritos al programa de Nefrored del Caribe Colombiano. La muestra se tomó por conveniencia, teniendo como referentes los pacientes que cumplían puntualmente las citas programadas para su valoración y seguimiento en el programa, constituida por 22 pacientes con Nefritis Lúpica, que consultan en la institución prestadora de salud la cual es centro de referencia en la atención de los pacientes en la ciudad de Barranquilla, de enero de 2017 a enero de 2018. Se tuvieron en cuenta los criterios de inclusión y exclusión, los cuales fueron; pacientes activos en el programa, que 
fueran de la región Caribe Colombiana, que no tuviesen otra patología asociada, y que de forma voluntaria participaran en el estudio.

Se excluyeron los pacientes con información insuficiente sobre las variables evaluadas, que consultaron ocasionalmente por esta enfermedad, menores de edad, y que no fueran de la región Caribe Colombiana.

Para la recolección de la información, se diseñó y validó un instrumento, tomando como referente teórico lo expuesto en la guía para el manejo de la enfermedad renal crónica y modelo de prevención y control de la enfermedad renal crónica en Colombia ${ }^{(11)}$.

Así mismo de las recomendaciones brindada por las guías Kidney Disease Improving Global Outcomes (KDIGO) 2014, en la cual se detallan complicaciones metabólicas, endocrinas y las dosificaciones detalladas de los tratamientos ${ }^{(12)}$. El instrumento tipo escala de Likert, estuvo conformada por las siguientes variables; características socio demográficas como: sexo, ubicación geográficas, estado civil, tiempo de diagnóstico de la enfermedad, y toma del medicamento ordenado. Para la validación del instrumento, se hizo una revisión por juicio de tres expertos y una prueba piloto con el fin de evaluar aspectos técnicos, mejorar y corregir las dificultades detectadas, mejorar los flujos de variables y el orden de las mismas.

Posteriormente se inició el proceso de recolección de la información aplicando el instrumento a los pacientes objeto de estudio. Seguidamente, se realizó la tabulación y el análisis de la información, para lo cual fue utilizado el programa estadístico SPSS (2013), donde se asentaron todas las variables a evaluar arrojando resultados exactos, sin sesgo de error. y al realizar el análisis se hizo en tablas de frecuencias y porcentajes. Los datos se analizaron con herramientas de estadística descriptiva y valores mínimos y máximos para las variables cuantitativas. El proyecto fue aprobado por el Comité de Ética de la institución prestadora de salud, los criterios éticos se fundamentaron según resolución 008430 del 4 octubre de 1993 la cual establece Normas científicas, técnicas y administrativas para investigación en salud, en Colombia ${ }^{(13)}$. La investigación se clasificó en categoría de investigación sin riesgo.

\section{RESULTADOS}

La población objeto de estudio estuvo conformada por 22 pacientes con diagnóstico de Nefritis Lúpica (NL) adscritos al programa de Nefrored del Caribe; el $96 \%$ de sexo femenino; en cuanto al grupo etareo se evidenció mayor representación en los grupos de menor o igual a 21 años (27,3\%), seguido por el 34 a 45 años $(27,3 \%) 22$ a 33 años $(22.7 \%)$ cada uno; se observó mayor procedencia de pacientes del departamento del Atlántico con 22 sujetos $(54,5 \%)$ residiendo en Barranquilla, seguido por Magdalena con 8 pacientes $(36,4 \%)$ procedentes del Municipio de Soledad y por último los Municipios de Baranoa y Sabanalarga que suman un $9 \%$ representándose con 2 pacientes; en cuanto al estado civil de los individuos, el $50,0 \%$ soltero, seguido de $36,4 \%$ casado, $4,5 \%$ separado, $4,5 \%$ unión libre y el $4.5 \%$ viudos.

Tabla 1. Datos Sociodemográficos de pacientes con Nefritis Lúpica.

\begin{tabular}{llrr}
\hline Datos Sociodemográficos & Indicadores & $\mathbf{n}$ & \% \\
\hline Sexo & Femenino & 21 & 96 \\
& Masculino & 1 & 4 \\
Grupo etareo & 21 años & 6 & 27,3 \\
& 22 a 33 años & 5 & 22,7 \\
& 34 a 45 años & 6 & 27,3 \\
Procedencia & 46 y más & 5 & 22,7 \\
& Barranquilla & 12 & 54,5 \\
& Soledad & 8 & 36,5 \\
Estado Civil & Otra (Sabanalarga, Baranoa) & 2 & 9,0 \\
& Soltero & 11 & 50 \\
& Casado & 8 & 36,4 \\
& Otro (unión libre, separado, viudo) & 3 & 13,5 \\
\hline
\end{tabular}

Fuente: Datos Obtenidos por los investigadores 
En cuanto al tiempo del diagnóstico de la enfermedad, se mantiene mayor porcentaje $72,7 \%$ los pacientes tienen diagnostico $\geq 2$ años, seguidos por pacientes con tiempo de diagnóstico entre 1 y 2 años por último los que tienen $<6$ meses de diagnóstico con un $9,1 \%$. Las respuestas dadas por la población sobre el cumplimiento en el horario para toma de medicamentos fueron que $86,4 \%$ cumplía con los horarios y solo un $13,6 \%$ no cumplía el horario (Tabla 2).

Tabla 2. Características de la enfermedad. $n=22$

\begin{tabular}{llll}
\hline Variable & Indicador & $\mathbf{n}$ & \multicolumn{1}{c}{$\%$} \\
\hline \multirow{3}{*}{ Tiempo de diagnóstico de la enfermedad } & $<6$ meses & 2 & 9,1 \\
& Entre 1 y 2 años & 4 & 18,2 \\
\multirow{2}{*}{ Toma usted el medicamento a la hora indicada } & $>2$ años & 16 & 72,7 \\
& Si & 19 & 86,4 \\
& No & 3 & 13,6 \\
\hline
\end{tabular}

Fuente: Datos Obtenidos por los investigadores

\section{DISCUSIÓN}

Se hallan condiciones socio demográficas de la población que la hacen vulnerable a la aparición de la enfermedad, además de su situación geográfica, por encontrarse ubicadas en la Región Caribe Colombiana, donde predominan pacientes con Lupus en Colombia. La muestra constituida de forma predominante por el sexo femenino en población adulta joven en edades promedios de 22-33 años, estos valores son afines a los vistos por Estrada ${ }^{(11)}$, el cual evidenció un promedio de edad similar a la población objeto de estudio del presente trabajo. La mitad es de estado civil soltera. La edad juega un rol importante en las personas con enfermedades crónicas como la $\mathrm{NL}$, aunque la esperanza de vida es cada día más alta, las diferentes organizaciones están trabajando para que los pacientes con esta enfermedad tengan mejor control y menos complicaciones ${ }^{(12,13)}$. En este contexto, existe una similitud con el trabajo(22) siendo que esta enfermedad es predominantemente femenina y su proporción varón: mujer. El estado civil de la población en estudio mostró en un porcentaje considerable que eran solteros, seguidos de los casados, observando diferencias estadísticamente significativas del grupo objeto de estudio y lo declarado por otros estudios ${ }^{(14,15)}$.

Con relación a la variable del tiempo de diagnóstico de la enfermedad, se logró evidenciar que un alto porcentaje $(72,7 \%)$ de la población de este estudio fue diagnosticado en un tiempo menor de 2 años, lo cual guarda relación con el estudio de Espinoza ${ }^{(16)}$. La demora en el diagnóstico siempre retrasa el inicio del tratamiento, lo cual coadyuva al aumento de la nefropatía, influyendo en los indicadores de morbilidad.

El lugar de procedencia fue mayoritariamente del departamento del Atlántico, teniendo como referente que el lugar de residencia de los pacientes objetos de estudio es la ciudad de Barranquilla, lo cual facilita su desplazamiento al programa de Nefrored del Caribe en la institución prestadora de salud objeto de estudio, lo cual guarda relación con lo expuesto por Marinho CLA ${ }^{(17,18)}$.

Otros estudios encontraron datos variables que entre el $64 \%$ y el $100 \%{ }^{(19)}$ de los pacientes toman medicamentos, lo cual contribuye a la adherencia. Este dato está estrechamente relacionado con el estudio realizado en el que la poblacion presenta un cumplimiento en el horario para toma de medicamentos, $86,4 \%$ manifestò cumplir con los horarios. Las fallas en el seguimiento a las prescripciones médicas en la toma de medicamentos en los pacientes cronicos conducen a la progresion de la enfermedad, lo que imposibilita que se realice un buen diagnóstico ${ }^{(20,21)}$. Dada la complejidad del comportamiento clínico de los pacientes con NL, se hace necesario en el futuro, estudios que discriminen las condiciones socio demográficas de los pacientes por la complejidad del diagnóstico y no permiten observar las diferencias que puedan presentarse, las cuales pueden ser relevantes para el inicio del tratamiento y la calidad de vida de los pacientes. 


\section{AGRADECIMIENTOS}

Este estudio se encuentra en el marco del proyecto de Nefro red del Caribe de la Clínica de la Costa en la Ciudad de Barranquilla, trabajo financiado por la Universidad Simón Bolívar. Se resalta la colaboración de los pacientes con Nefritis Lúpica que se encuentran adscrito al programa de Nefro red del Caribe.

\section{CONFLICTOS DE INTERÉS}

Los autores declaran no tener ningún conflicto de interés.

\section{REFERENCIAS BIBLIOGRÁFICAS}

1. Zamorano JL, García Moll X, Ferrari R, Greenlaw N. Características demográficas y clínicas de los pacientes con enfermedad coronaria estable: resultados del registro CLARIFY en España. Rev. Esp Cardiol. [revista en internet] 2014 [30 de abril de 2018] 67 (7) Disponible en: http://www.revespcardiol.org/es/caracteristi cas-demograficas-clinicas-lospacientes/articulo/90332424/

2. Oliveros D, Arrieta M, Cajigas E, Gonima Valero E. Características clínicas de los pacientes que consultan a la clínica de dolor crónico del hospital militar central, histórico de 20 años. Revista Med. [revista en internet] 2011 [30 de junio de 2018] 19 (1), 102-111. Disponible: http://www.redalyc.org/pdf/910/910225340 12.pdf

3. Bermúdez Marrero WB, Vizcaíno Luna $Y$, Fusté Jiménez C, González Otero Z A, Egües Mesa JL. Caracterización clínico epidemiológica de pacientes con lupus eritematoso sistémico. Hospital Universitario Arnaldo Milán Castro. Rev Cuba Reumatol [revista en internet] 18 (I) 2016 [30 de abril de 2018] Disponible en: http://scielo.sld.cu/scielo.php?script=sci_art text\&pid $=$ S1817-59962016000400003

4. Willrich Böell JE, Guerreiro Vieira da Silva DM, Hegadoren KM. Factores socio demográficos y condicionantes de salud asociados a la resiliencia de personas con enfermedades crónicas: un estudio transversal. Rev. Latino-Am. Enfermagem [revista en internet] 2016; [30 de octubre de 2017] 24:e2786. Disponible en: http://www.redalyc.org/pdf/2814/28144972 7026.pdf

5. Cantú G, Rodríguez G, Luque-Coqui $M$, Romero $B$, Valverde $S$, Vargas $S$, ReyesLópez A, Medeiros M. Análisis de las características socio demográficas de los pacientes con enfermedad renal crónica terminal: diferencias en un periodo de seis años. Bol Med Hosp Infant Mex 2012;69(4):290-294 [Revista en Internet] Rev. Bol Med Hosp Infant Mex. N4. México, 2012. [consultado el 30 de octubre de 2016] Disponible en: https://www.researchgate.net/publication/2 62464103_Analisis_de_las_caracteristicas_s ociodemograficas_de_los_pacientes_con_enf ermedad_renal_cronica_terminal_diferencias _en_un_periodo_de_seis_anos
6. Calvo AJ, Silva-Fernández $\mathrm{L}$, Ucar Angulo $\mathrm{E}$, Pego Reigosa JM, Olivé A, MartínezFernández C, Martínez-Taboada V, Marenco JL, Lozai E, López-Longo J, Gómez-Reinok JJ, Galindo-Izquierdo M, Fernández-Nebro A, Cuadrado MJ, Aguirre-Zamorano MA, ZeaMendoza A, Rúa-Figueroa I. Consenso de la Sociedad Española de Reumatología sobre el uso de terapias biológicas en el lupus eritematoso sistémico. Rev. Reumatol Clin. [revista en internet] 9 (5) 2013 281-96 [consultado el 6 de Agosto de 2018]. Disponible:

http://www.reumatologiaclinica.org/es/cons enso-sociedad-espanola-reumatologiasobre/articulo/S1699258X13000922/

7. Rodríguez García AN, Osorio Sagrero $\mathrm{SB}$, Maldonado Velázquez MR, Faugier Fuentes E. Lupus eritematoso generalizado juvenil: patrones clínicos e inmunológicos en una cohorte de 150 pacientes del Hospital Infantil de México. An Med (Mex) [revista en internet] 2016; [consultado el 30 de octubre de 2017] 61 (3): 182-187 Disponible en: http://www.medigraphic.com/pdfs/abc/bc2016/bc163e.pdf

8. Estévez del Toro MH, Chico Capote A, Sánchez Bruzón Y, Gutiérrez Rojas Á, Chong López A. Factores predictores de la respuesta a la terapia de inducción con ciclofosfamida en Nefritis Lúpica proliferativa. Rev cubana med [revista en internet] 46 (2) Ciudad de la Habana jul.sep. 2007 [consultado el 30 de julio de 2018]. Disponible: http://scielo.sld.cu/scielo.php?script=sci_art text\&pid $=$ S0034-75232007000200004

9. Prieto $F$, Cabañas C, Villagra V. Características de los pacientes en espera de trasplante renal. Rev. Mem. Inst. Investig. Cienc. Salud [revista en internet], 13(1) Abril 2015: 49-57 [consultado el 30 de octubre de 2017]. Disponible en: http://scielo.iics.una.py/pdf/iics/v13n1/v13n $1 \mathrm{a08} . \mathrm{pdf}$

10. Kasamatsu E. Creación del Registro de Glomerulopatias del Paraguay.Rev. Mem. Inst. Investig. Cienc. Salud, [revista en internet] 3 (1), 2005[consultado el 30 de octubre de 2017]. Disponible en: http://scielo.iics.una.py/pdf/iics/v3n1/v3n1 a20.pdf

11. Ministerio de la Protección social. Guía de práctica clínica para el diagnóstico y 
tratamiento de la enfermedad renal crónica. [monografía en internet] $\mathrm{N}^{\circ}$. 59. Colombia 2016. [consultado el 30 de octubre de 2017] Disponible: http://gpc.minsalud.gov.co/gpc_sites/Repo sitorio/Otros_conv/GPC_e_renal/GPC_enfer medad_renal_adopcion.pdf

12. Gorostidi M, Santamaría $R$, Alcázar $R$, Fernández-Fresnedo G, Galcerán JM, Goicoechea $M$, et al. Documento de la Sociedad Española de Nefrología sobre las guías KDIGO para la evaluación y el tratamiento de la enfermedad renal crónica. Rev. Nefrología (Madr.) [revista en internet] 34 (3) Cantabria 2014 [consultado el 30 de octubre de 2017] Disponible en: http://scielo.isciii.es/scielo.php?script $=$ sci_ arttext\&pid=S0211-69952014000300005

13. Ministerio de la protección social. Resolución 008430 del 4 octubre de 1993 la cual establece Normas científicas, técnicas y administrativas para investigación en salud, en Colombia 1993 [monografía en internet] [consultado el 30 de octubre de 2018] Disponible: https://www.minsalud.gov.co/sites/rid/List s/BibliotecaDigital/RIDE/DE/DIJ/RESOLUCI ON-8430-DE-1993.PDF

14. Estrada Vidal J, Pasalodos Norkis, Pila Pérez R, Ramírez Rodríguez L, Gallardo Roca L. Tratamiento de la nefritis lúpica grado IV con bolos de ciclofosfamida. AMC [Internet]. 2004 Oct [citado 2018 Ago 03]; 8(5): 10-21. Disponible en: http://scielo.sld.cu/scielo.php?script=sci_a rttext\&pid $=S 1025$ 02552004000500002\&lng =es.

15. Barrera Ortiz L, Vargas Rosero E, Cendales PA. Encuesta de caracterización para el cuidado de una persona con enfermedad crónica. Investig Enferm. Imagen Desarr. [revista en internet] 2015 17(1):27-43. [consultado el 30 de octubre de 2017] Disponible en: http://dx.doi.org/10.11144/Javeriana.IE17 -1 .eccp

16. Pinto Afanador N. La cronicidad y el cuidado familiar, un problema de todas las edades: los cuidadores de adultos. Avances en Enfermería [revista en internet] 2004 22(1), 54-60. [consultado el 30 de octubre de 2017] Disponible en: https://revistas.unal.edu.co/index.php/ avenferm/article/view/37941

17. Moya Ruiz MA. Estudio del estado emocional de los pacientes en hemodiálisis. Enferm Nefrol Madrid [revista en internet] 20 (1) ene./mar. 2017 [consultado 1 de noviembre de 2017]. Disponible en: http://scielo.isciii.es/scielo.php?script=sci arttext\&pid=S2254-28842017000100007

18. Vinaccia S, Quiceno Japcy M, Medina A, Castañeda B, Castelblanco P. Calidad de vida. Resiliencia, Percepción y conducta de
Enfermedad en Pacientes con Diagnóstico de Dispepsia Funcional. Rev. Acta Colombiana de Psicología [revista en internet] 17 1. 2014. [consultado el 2 de noviembre de 2017] Disponible en : http://www.redalyc.org/pdf/798/79831197 007.pdf

19. Espinoza Cuevas G, Nathaly Vegonia Calcina Cuevas, Yercin Mamani Ortiz, Maritza Jiménez de Sánchez, José Enrique Gutiérrez Mendez. Características clínicoepidemiológicas de la nefritis lúpica en pacientes con lupus en el Complejo Hospitalario Viedma, Gestión 2010- 2015. Rev Cient Cienc Méd) 18 (2) Cochabamba 2015 [revista en internet][contultado el 30 de octubre de 2017]. Disponible en: http://www.scielo.org.bo/scielo.php?script $=$ sci_arttext $\&$ pid $=$ S181774332015000200002

20. Montalvo Prieto A, Flórez Torres IE. Cuidadores de Cartagena y su contexto de cuidado: Estudio comparativo. Rev. Aquichan [Revista en Internet] $\mathrm{N}^{\circ} 2$. Colombia, 2009. Disponible en: http://aquichan.unisabana.edu.co/index.ph p/aquichan/article/view/1477/1677

21. Alencar Marinho CL, Freire de Oliveira J, da Silva Borges JE, Cavalcante EF, Fernandes V; Souza da Silva R. Associação entre características socio demográfico e qualidade de vida de pacientes renais crônicos em hemodiálise. Rev. Cuid. [revista en internet] 1. Colombia, 2018 [consultado el 5 de julio de 2018]. Disponible en: https://www.revistacuidarte.org/index.php /cuidarte/article/view/483/902

22. Pinto P. LF, Velásquez F. CJ, Márquez H. J. Subgrupos de Lupus Eritematoso Sistémico: influencia de la edad de inicio, la raza, el sexo y el perfil de anticuerpos en las manifestaciones clínicas de la enfermedad. Rev Colomb Reumatol [revista en internet] 15(4) Bogotá Oct./Dic. 2008 [consultado el 6 de agosto de 2018] Disponible en: http://www.scielo.org.co/scielo.php?script =sci_arttext\&pid $=$ S0121$81232008000400004 \% 20$ 